\title{
Relationship between splenomegaly and hematologic findings in patients with hepatosplenic schistosomiasis
}

\author{
Luiz Arthur Calheiros Leite \\ Ana Lúcia Coutinho Domingues \\ Edmundo Pessoa Lopes \\ Rita de Cássia dos Santos Ferreira \\ Adenor de Almeida Pimenta Filho \\ Caíque Silveira Martins da Fonseca \\ Bianka Santana dos Santos \\ Vera Lúcia de Menezes Lima
}

Universidade Federal de Pernambuco - UFPE, Recife, PE, Brazil
Conflict-of-interest disclosure:

The authors declare no competing financial interest

Submitted: 9/26/2012

Accepted: 3/14/2013

\section{Corresponding author:}

Luiz Arthur Calheiros Leite

Universidade Federal de Pernambuco - UFPE

Departamento de Bioquímica - Centro de

Ciências Biológicas - CCB

Avenida Professor Moraes Rego, S/N, Cidade

Universitária

50640-420 Recife, PE, Brazil

lahemato@hotmail.com
Background: Schistosomiasis is a tropical disease. Patients who develop hepatosplenic schistosomiasis have clinical findings including periportal fibrosis, portal hypertension, cytopenia, splenomegaly and gastrointestinal hemorrhage.

Objective: The aim of this study was to analyze the hemostatic and hematologic findings of patients with schistosomiasis and correlate these to the size of the spleen.

Methods: Fifty-five adults with hepatosplenic schistosomiasis and 30 healthy subjects were selected through a history of contact with contaminated water, physical examination and ultrasound characteristics such as periportal fibrosis and splenomegaly in the Gastroenterology Service of the Universidade Federal de Pernambuco. Blood samples were collected to determine liver function, blood counts, prothrombin (international normalized ratio), partial thromboplastin time and fibrinogen and D-Dimer levels using the Pentra 120 hematological analyzer (HORIBA/ABX), Density Plus (test photo-optical Trinity Biotech, Ireland) and COBAS analyzer 6000 (Roche). Furthermore, the longitudinal size of the spleen was measured by ultrasound (Acuson X analyzer 150, Siemens). The Student t-test, the Fisher test and Pearson's correlation were used to analyze the results with statistical significance being set for a p-value $<0.05$.

Results: The mean age was higher for the Study Group than for the Control Group (54 \pm 13.9 vs. $38 \pm 12.7$ years). The average longitudinal diameter of the spleen was $16.9 \mathrm{~cm}$ (Range: 12.3-26.3 cm). Anemia is a common finding in patients with schistosomiasis (36.3\%). The mean platelet and leukocyte counts of patients were lower than for the Control Group ( $p$-value $<0.001)$. Moreover, the international normalized ratio $(1.42$ vs. 1.04), partial thromboplastin time (37.9 vs. 30.5 seconds) and D-Dimer concentration (393 vs. $86.5 \mathrm{ng} / \mathrm{mL}$ ) were higher for the Study Group compared to the Control Group.

Conclusion: This study suggests that hematological and hemostatic abnormalities are associated with splenomegaly, hypersplenism and portal hypertension.

Keywords: Schistosomiasis mansoni; Schistosomiasis; Splenomegaly; Hypersplenism; Thrombocytopenia

\section{Introduction}

Schistosomiasis mansoni is a chronic parasitic disease and the most prevalent tropical liver disease in the northeastern region of Brazil. There are 200 million people affected by Schistosoma mansoni worldwide with 600 million people being exposed. Around 5-7\% of the patients infected by $S$. mansoni progress to the most severe form, hepatosplenic schistosomiasis (HS). Many of these patients exhibit high morbidity associated with periportal fibrosis, portal hypertension, splenomegaly, upper digestive tract bleeding and cytopenia ${ }^{(1-5)}$.

Hypersplenism is a consequence of massive splenomegaly and is a common finding in chronic liver diseases. In schistosomiasis, this results from hyperplasia of the reticuloendothelial system and consequently venous congestion caused by portal hypertension. Studies have reported a correlation between the increase of spleen size and drops in blood cell counts, mainly the platelet count. These findings depend on the severity of portal hypertension as some studies have shown that thrombocytopenia is more common in HS patients, especially after episodes of digestive tract bleeding ${ }^{(6-8)}$.

Some studies have reported that compensated HS patients exhibit normal hepatic function even with some abnormalities in blood clotting. Other reports have called attention to hemostatic abnormalities in decompensated HS patients who develop ascites and upper digestive tract bleeding including increases in the prothrombin time/international normalization ratio (PT/INR), partial thromboplastin time (PTT), thrombin time (TT) and abnormalities in K-dependent factors. The liver plays an important role in the control of blood coagulation and disorders of parenchymal liver cell function affect the hemostatic system. Liver disorders may be associated with the reduction of coagulation protein synthesis, in particular in cirrhosis patients. These findings are still unclear and represent a contradiction for medical research, especially in studies involving liver diseases such as cirrhosis and schistosomiasis and other liver disorders that lead to progressive hepatic damage ${ }^{(9-11)}$.

The purpose of this study was to evaluate the hematological and hemostatic abnormalities in patients with severe forms of schistosomiasis, and possible associations with splenomegaly and portal hypertension. 


\section{Methods}

\section{Diagnosis}

A prospective study was undertaken involving 55 compensated HS patients previously treated using praziquantel $(50 \mathrm{mg} / \mathrm{kg})$ and 30 healthy subjects in the Outpatient Gastrointestinal Service of Hospital das Clínicas of the Universidade Federal de Pernambuco (UFPE), Recife, Brazil from 2010 to 2012. Patients and controls had comparable socioeconomic conditions including similar salaries, educations and lifestyles. The diagnosis and consecutive selection of HS patients was based on clinical history, physical examination and an abdominal ultrasonography that showed periportal fibrosis and splenomegaly. The abdominal ultrasound was performed using an ultrasound analyzer (Acuson X 150, 3.5 $\mathrm{MHz}$, Siemens). For this, we used the Cairo and Niamey protocols to measure the longitudinal diameter of the spleen and classify the pattern of fibrosis as central fibrosis (Pattern D), advanced fibrosis (Pattern E) or very advanced fibrosis (Pattern F) ${ }^{(12-14)}$.

Upper digestive tract endoscopy was used either to confirm or exclude the presence of esophageal varices and viral marker and liver function tests were performed to exclude viral hepatitis B and C. Patients suffering from alcoholism ( $>60 \mathrm{~g}$ /day for men and $>40$ $\mathrm{g} /$ day for women) ${ }^{(15)}$, systemic diseases such as diabetes mellitus, collagenosis, blood diseases (lymphoproliferative disease and lymphomas), those that were taking hepatotoxic, antiaggregant or anticoagulant drugs and every patient with a history of splenectomy or blood transfusions (within the previous 3 months) were carefully excluded. Stool samples were examined to detect intestinal parasites by the Lutz-Hoffman followed by Kato-Katz method. Routine liver tests included analysis of aspartate transaminase (AST) alanine transaminase (ALT), alkaline phosphatase (ALP), gamma-glutamyl transferase $(\gamma \mathrm{GT})$ and albumin. The Control Group had the same exclusion criteria as described above and were checked for possible contact with water contaminated by the cercariae of S. mansoni and positive parasitological tests. The compensated hepatosplenic form corresponds to the severe form of disease and includes patients with hepatosplenomegaly and portal hypertension, but without ascites, jaundice or encephalopathy ${ }^{(9)}$.

\section{Sample collection}

About $15 \mathrm{~mL}$ of venous blood was drawn under aseptic conditions without stasis and placed in vacuum tubes (Vacutainer; Becton Dickinson, UK). The first blood sample was placed in a polypropylene tube containing $0.106 \mathrm{M}$ trisodium citrate at a bloodanticoagulant ratio of 9:1. These tubes were used for the coagulation tests (PT, PTT, fibrinogen and D-dimer). The blood samples were centrifuged at $2000 \mathrm{~g}$ for 15 minutes at $4^{\circ} \mathrm{C}$. Subsequently, the platelet free plasma was quickly distributed in $0.5 \mathrm{~mL}$ aliquots in plasticcapped tubes and stored at $-80^{\circ} \mathrm{C}$ for six months until processing. A second sample, placed in a tube containing ethylenediaminetetraacetic acid (EDTA), was used for the complete blood count (CBC). A blood smear was prepared and stained for conventional microscopic analysis. The $\mathrm{CBC}$ was carried out by electrical impedance or light dispersion using a Pentra 120 analyzer (ABX, São Paulo, SP, Brazil). A third sample was collected for routine liver tests (AST,
ALT, $\gamma \mathrm{GT}$, ALP, albumin) using biochemistry tubes (Becton Dickinson, UK) and the assay was performed using the COBAS 6000 analyzer (Roche). The aliquots of plasma serum samples were also stored for the other tests. The hemostatic tests (PT/INR, PTT, fibrinogen and D-dimer) were carried out in an automated photooptical coagulometer, (Trinity Biotech, Acton, Ireland) following the manufacturer's instructions. The study was conducted according to the norms of the Declaration of Helsinki. The protocols for the collection and use of human samples were submitted and approved by the Research Ethics Committee of the Health Sciences Center of the UFPE ( $\left.N^{\circ} 028 / 11\right)$. All subjects received an explanation about the study and signed informed consent forms.

\section{Statistical analysis}

Statistical analyzes were performed using the unpaired Student t-test and Fisher's test for contingency analysis. These analyzes were performed using the Statistical Package for Social Sciences software (SPSS 17.0, Chicago, IL, USA). Continuous variables were expressed as means \pm standard deviation (SD), median and range. Furthermore, the Pearson correlation test was employed to examine the relationship between the longitudinal diameter of the spleen and platelet counts. P-values of less than 0.05 were considered statistically significant.

\section{Results}

The results are shown in Tables $1 \& 2$ and in Figures 1 to 4 . The patients had a mean age of $54 \pm 13.9$ years and the 30 control subjects had a mean age of $38.3 \pm 13.7$ years $(p$-value $<0.001)$ but no difference was found in respect to the gender. The Niamey

Table 1 - Liver function tests in hepatosplenic schistosomiasis patients and the Control Group

\begin{tabular}{lrrr}
\hline Variable & $\begin{array}{c}\text { Control } \\
\text { Group }\end{array}$ & $\begin{array}{c}\text { Hepatosplenic } \\
\text { Group }\end{array}$ & p-value \\
\hline Participants & 30 & 55 & \\
AST (units/L) median (range) & $20(15-29)$ & $39(15-201)$ & $<0.0001$ \\
ALT (IU/L) median (range) & $17.5(9-27)$ & $36(12-257)$ & $<0.0001$ \\
ALP (IU/L) median (range) & $67.0(28-105)$ & $132(58-1321)$ & $<0.0001$ \\
VGT (IU/L) median (range) & $24.5(9-75)$ & $93(16-756)$ & $<0.0001$ \\
Albumin (g/L) (mean \pm SD) & $43 \pm 5$ & $38 \pm 6$ & $<0.0001$ \\
\hline
\end{tabular}

AST: Aspartate transaminase; ALT: Alanine transaminase; ALP: Alkaline phosphatase; $\gamma \mathrm{GT}$ : gamma-glutamyl transferase

Table 2 - Distribution of the hepatosplenic schistosomiasis patients according to type of cytopenia

\begin{tabular}{lrc}
\hline Cytopenias & $\mathbf{n}$ & $\mathbf{\%}$ \\
\hline No cytopenia & 12 & 21.8 \\
Leukopenia in isolation & 4 & 7.27 \\
Thrombocytopenia in isolation & 12 & 21.8 \\
Bicytopenia & 17 & 30.9 \\
Pancytopenia & 10 & 18.1 \\
Total & 55 & 100 \\
\hline
\end{tabular}




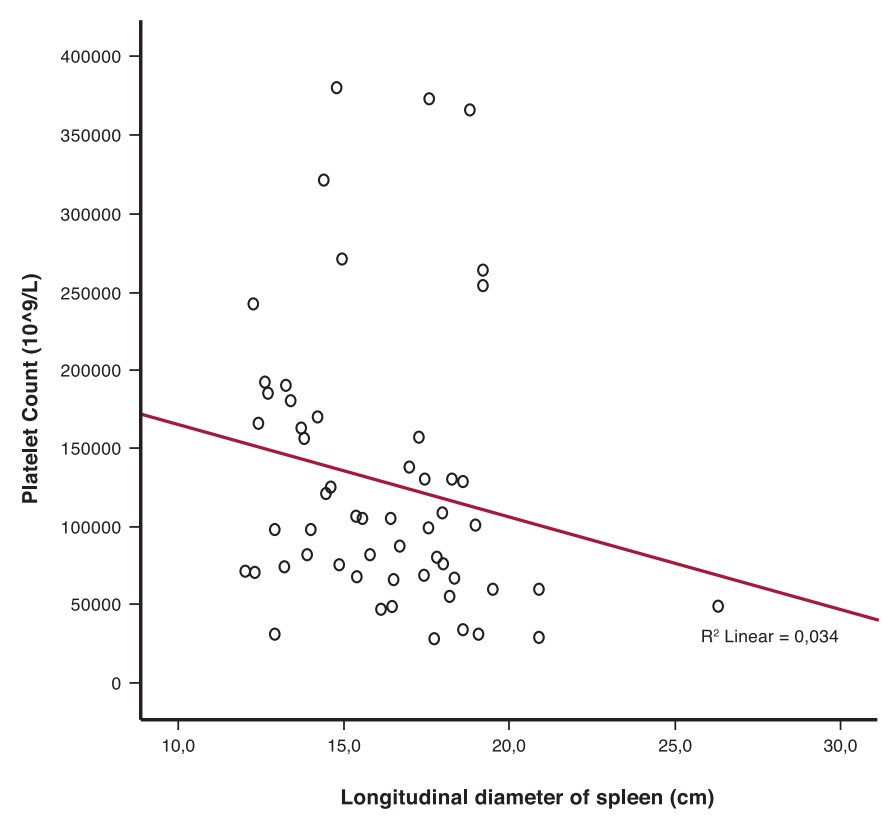

Figure 1 - Relationship between longitudinal diameter of the spleen and platelet counts in hepatosplenic patients

A direct positive correlation was observed between splenomegaly and platelet counts in schistosomiasis patients $(\mathrm{r} 2=0.034 ; \mathrm{p}$-value $=0.02)$
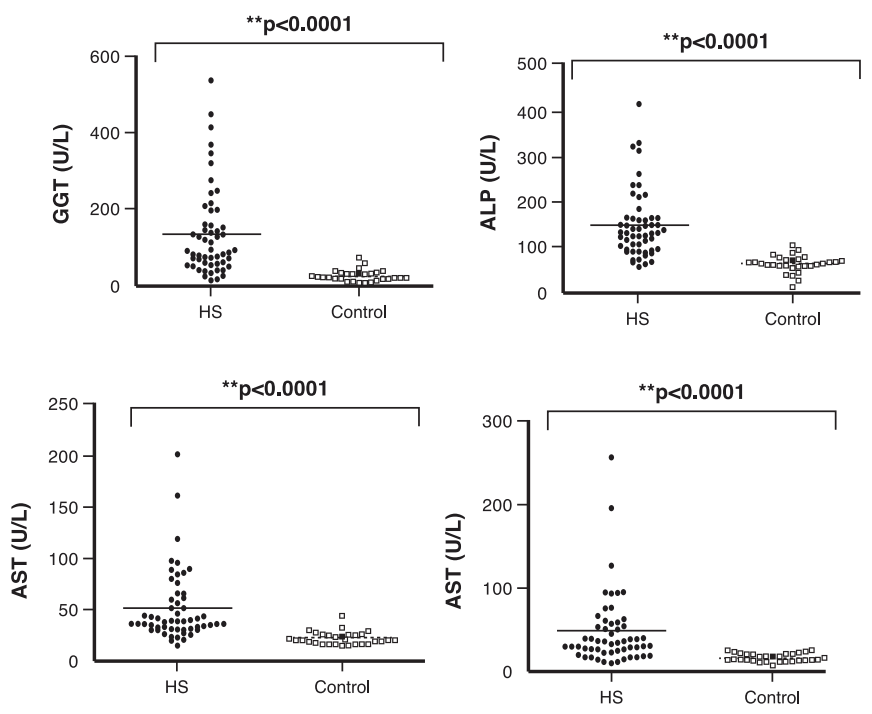

Figure 3 - Routine liver tests in the Control and Schistosomiasis Groups

Data are expressed as means. Controls vs. hepatosplenic schistosomiasis (HS) patients AST: Aspartate transaminase; ALT: Alanine transaminase; $\gamma$ GT: gamma-glutamyl transferase

classification of fibrosis showed predominance of advanced (pattern E - 30 patients; $54.5 \%$ ), followed by pattern D (17 patients; $30.9 \%$ ) with only eight patients (14.5\%) having very advanced fibrosis (pattern F). The routine liver tests showed significant differences in relation to parameters such as albumin, AST, ALT, ALP and $\gamma \mathrm{GT}$ between the groups (p-value $<0.001$; Figure 1).

Upper gastrointestinal bleeding was found in $34(61.8 \%)$ patients. The mean longitudinal diameter of the spleen of the

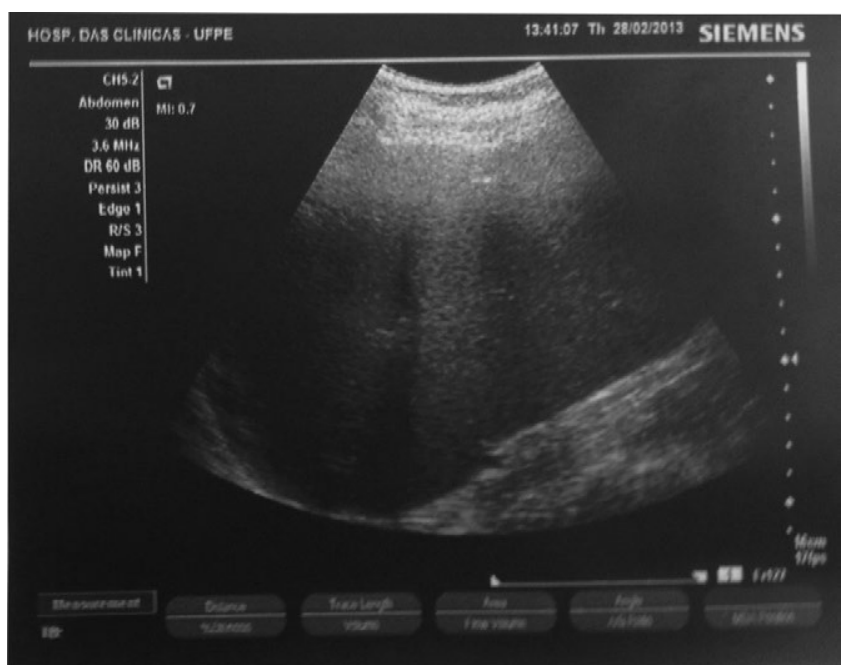

Figure 2 - Image showing a massive splenomegaly $(18.7 \mathrm{~cm})$ in a hepatosplenic schistosomiasis patient
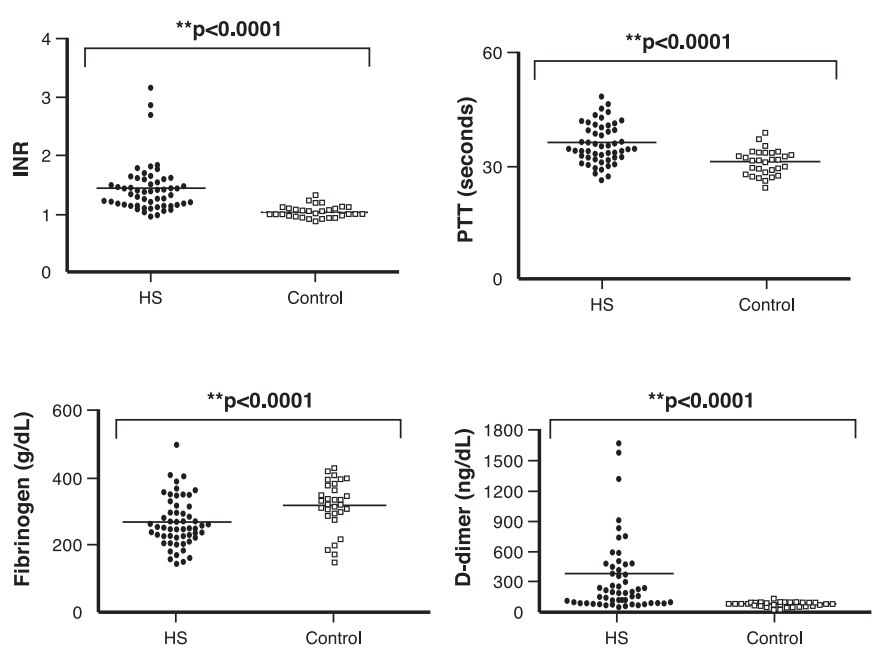

Figure 4-Coagulation and fibrinolytic parameters in Control and Schistosomiasis Groups

Data are expressed as mean

Controls vs. hepatosplenic schistosomiasis (HS) patients

IRN: international normalized ratio; PTT: partial thromboplastin time;

The D-dimer concentration was used for measure the fibrinolytic status and risk of bleeding

55 patients was $16.9 \mathrm{~cm}$ (Range: $12.3-26.3 \mathrm{~cm}$ ) and the median platelet count was $101.0 \times 10^{9} / \mathrm{L}$. A total of $38(69.1 \%)$ patients had thrombocytopenia (platelet counts below $150 \times 10^{9} / \mathrm{L}$ ). Furthermore, $14.5 \%$ of patients had platelet counts below $50 \mathrm{x}$ $10^{9} / \mathrm{L}$ (Table 1). There was an inverse correlation between the longitudinal diameter of the spleen and the platelet count (Figure 2 ). It was also found that $36.3 \%$ of patients had anemia and $47.3 \%$ presented leukopenia (cytopenias were different in each 
patient). Table 2 shows the distribution of HS patients according to the type of cytopenia. Figure 3 demonstrates the massive splenomegaly of one patient with schistosomiasis.

Figure 4 demonstrates that HS patients presented with increased INR, PTT, and fibrinogen and D-dimer concentrations. There were significant differences in respect to these parameters on comparing the Control Group with patients (p-value $<0.001$ ).

\section{Discussion}

Portalhypertension is one of the mostimportant consequences of $S$. mansoni infections. Due to the fibrotic process and venous congestion, HS patients develop hemodynamic changes associated with splenomegaly and high morbidity rates ${ }^{(16)}$. In a recently study in the same region, Dias et al. ${ }^{(17)}$ showed that $61 \%$ of HS patients had advanced or very advanced periportal fibrosis. In the current study, all HS patients presented with a severe form of HS and portal hypertension, 38 (69\%) had advanced and very advanced liver fibrosis (pattern E+F) and 34 (61.8\%) patients had gastrointestinal bleeding. This proves that these patients have advanced disease and that changes in liver function tests may be related to the high frequency of advanced fibrosis.

Camacho-Lobato et al. and Tanabe reported that a large number of HS patients present conserved liver function even with periportal fibrosis ${ }^{(9,11)}$. However, this study demonstrates elevated liver function tests and remarkable changes in coagulation tests. Some studies have reported varied frequencies of thrombocytopenia (30 to $75 \%$ ) depending on the stage of portal hypertension ${ }^{(8-16)}$. Lower values of platelet counts are seen especially in patients with HS after gastrointestinal bleeding. This was also seen in the current work with the possible cause being the increase of spleen and hypersplenism leading to frequent thrombocytopenia, leukopenia and anemia.

Thrombocytopenia is a common feature in chronic liver disease. Correia et al. ${ }^{(8)}$ showed that thrombocytopenia can be compensated by increased levels of von Willebrand factor in HS patients. Other studies have shown that nearly $50 \%$ of HS patients exhibit thrombocytopenia, $16 \%$ bicytopenia and $7 \%$ anemia or leukopenia ${ }^{(18)}$. Anemia in patients with liver disease is often multifactorial and may be associated with iron and folic acid deficiency or even an inflammatory process. Red blood cell survival is often shortened in chronic liver disease with the increased spleen being a major site of red blood cell destruction ${ }^{(17)}$. In this study, high frequencies of thrombocytopenia (74.1\%), leukopenia $(47.3 \%)$, anemia $(36.3 \%)$, bicytopenia $(30.9 \%)$ and pancytopenia (18.2\%) were found. The high frequencies of cytopenias found may be associated with the increased spleen size and the consequent hypersplenism leading to an increase in scavenging and retention of blood cells in the spleen, mainly platelets and white blood cells. Furthermore, anemia may be accentuated because of the low socioeconomic situation of the patients and due to blood loss from upper digestive tract bleeding.

Berzigotti et al. ${ }^{(19)}$ used the diameter of the spleen combined with platelet count as a noninvasive marker of portal hypertension; these tools can also be used to predict esophageal varices and upper gastrointestinal bleeding episodes, especially in asymptomatic patients with cirrhosis. In this study, an inverse relationship was found between platelet count and longitudinal diameter of the spleen. Concomitant enlargement of the spleen from 15 to $20 \mathrm{~cm}$ together with a platelet count below $100 \mathrm{x}$ $10^{9} / \mathrm{L}$ is an excellent predictor of portal hypertension, esophageal varices and upper digestive tract bleeding, even in asymptomatic patients with schistosomiasis. These two parameters combined with an increased INR could be a useful noninvasive tool to predict a different behavior of portal hypertension in patients with HS. Nevertheless, further studies are needed to identify other noninvasive markers and thus validate a score to classify the different stages of schistosomiasis and other liver diseases.

A recent study showed that the levels of thrombopoietin and reticulated platelets were normal in schistosomiasis patients with portal hypertension and that the bone marrow produces platelets normally ${ }^{(20)}$. The cytopenias in schistosomiasis patients may occur because the cells are retained in the spleen due to difficulty in draining portal blood. Additionally, platelets may be retained in the sinusoidal spaces of the fibrotic liver. Some studies have shown that the degree of liver fibrosis is associated with the degree of esophageal varices and that the risk of bleeding is associated with the degree of liver fibrosis ${ }^{(21)}$. Studies by KöpkeAguiar et al. ${ }^{(20)}$ report that there are no changes in the production of platelets in the bone marrow in schistosomiasis patients.

\section{Conclusion}

The present study suggests that the hematological abnormalities seen in HS patients are associated with splenomegaly, hypersplenism and portal hypertension. However, further studies are needed to verify whether the platelet count might be a non-invasive tool to assess portal hypertension.

Financial support: Conselho Nacional de Desenvolvimento Científico e Tecnológico, Fundação de Amparo à Ciência e Tecnologia de Pernambuco, Coordenação de Aperfeiçoamento de Pessoal de Nível Superior

\section{References}

1. Ross AG, Bartley PB, Sleigh AC, Olds GR, Li Y, Williams GM, et al. Schistosomiasis. N Engl J Med. 2002;346(16):1212-20. Comment in: N Engl J Med. 2002;347(10):766-8; author reply 766-8.

2. Gryseels B, Polman K, Clerinx J, Kestens L. Human schistosomiasis. Lancet. 2006; 368(9541):1106-18.

3. Coutinho EM, Abath FG, Barbosa CS, Domingues AL, Melo MC, Montenegro SM, et al. Factors involved in Schistosoma mansoni infection in rural areas of northeast Brazil. Mem Inst Oswaldo Cruz. 1997;92(5):707-15.

4. Silva CA, Oliveira KF, Carvalho VC, Domingues AL, Brandt CT, Lima VL. Surgical treatment effect on the liver lecitin: cholesterol acyltransferase (LCAT) in schistosomiasis mansoni. Acta Cir Bras. 2001;17(Supl. 1):28-30.

5. Ferraz AA, Albuquerque PC, Lopes EP, Araújo Jr JG, Carvalho AH, Ferraz EM. The influence of periportal (pipestem) fibrosis on long term results of surgical treatment for schistosomotic portal hypertension. Arq Gastroenterol. 2003;40(1):4-10. 
6. McCormick PA, Murphy KM. Splenomegaly, hypersplenism and coagulation abnormalities in liver disease. Baillieres Best Pract Res Clin Gastroenterol. 2000;14(6): 1009-31.

7. Bosch J, Abraldes JG, Berzigotti A, García-Pagán JC. Portal hypertension and gastrointestinal bleeding. Semin Liver Dis. 2008;28(1):3-25.

8. Correia MC, Domingues AL, Lacerda HR, Santos EM, Machado CG, Hora V, et al. Platelet function and the von Willebrand factor antigen in the hepatosplenic form of schistosomiasis mansoni. Trans R Soc Trop Med Hyg. 2009;103(10):1053-8.

9. Camacho-Lobato L, Borges DR. Early liver dysfunction in schistosomiasis. J Hepatol. 1998;29(2):233-40.

10. Omran SA, Amin HM, el-Bassiouni NE, Essawy FM, Toiema SM. Vitamin $\mathrm{K}$ dependent coagulation proteins in endemic hepatosplenomegaly in Egypt. J Clin Pathol. 1994;47(6):502-4.

11. Tanabe M. Haemostatic abnormalities in hepatosplenic schistosomiasis mansoni. Parasitol Int. 2003;52(4):351-9.

12. Richter J, Hatz C, Campagne G, Bergquist NR, Jenkins JM; UNDP/ World Bank/WHO Special Programme for Research and Training in Tropical Diseases. Ultrasound in schistosomiasis: a practical guide to the standardized use of ultrasonography for the assessment of schistosomiasis-related morbidity: Second International Workshop. October 22 - 26, 1996, Niamey, Niger.

13. Richter J, Domingues AL, Barata CH, Prata AR, Lambertucci JR. Report of the second satellite symposium on ultrasound in schistosomiasis. Mem Inst Oswaldo Cruz. 2001;96(Suppl):151-6.
14. Domingues AL, Lima AR, Dias HS, Leao GC, Coutinho A. An ultrasonographic study of liver fibrosis in patients infected with Schistosoma mansoni in north-east Brazil. Trans R Soc Trop Med Hyg. 1993;87(5):555-8.

15. Martins RD, Borges DR. Ethanol challenge in nonalcoholic patients with schistosomiasis. J Clin Pathol. 1993;46(3):250-3.

16. Maia MD, Lopes EP, Ferraz AA, Barros FM, Domingues AL, Ferraz EM. Evaluation of splenomegaly in the hepatosplenic form of mansonic schistosomiasis. Acta Trop. 2007;101(3):183-6.

17. Dias HS, Domingues AL, Cordeiro FT, Jucá N, Lopes EP. Associating portal congestive gastropathy and hepatic fibrosis in hepatosplenic mansoni schistosomiasis. Acta Trop. 2013;126(3):240-3.

18. Petroianu A, Oliveira AE, Alberti LR. "Hiperesplenism" in portal hypertension provoked by Manson's schistosomiasis. Rev Bras Hematol Hemoter. 2004;26(3):195-201.

19. Berzigotti A, Seijo S, Arena U, Abraldes JG, Vizzutti F, García-Pagán JC, et al. Elastography, spleen size, and platelet count identify portal hypertension in patients with compensated cirrhosis. Gastroenterology. 2013;144(1):102-11. Comment in: Gastroenterology. 2013;144(1):19-22.

20. Köpke-Aguiar LA, de Leon CP, Shigueoka DC, Lourenço DM, Kouyomdjian M, Borges DR. Reticulated platelets and thrombopoetin in schistosomiasis patients. Int J Lab Hematol. 2009;31(1):69-73.

21. Andrade ZA. Schistosomiasis and hepatic fibrosis regression. Acta Trop. 2008;108(2-3):79-82. 